\title{
Diverse mechanisms for inflammasome sensing of cytosolic bacteria and bacterial virulence
}

\author{
Yue Zhao and Feng Shao
}

\section{Addresses}

National Institute of Biological Sciences, Beijing, 102206, China;

Corresponding author:

Feng Shao, Ph.D.

National Institute of Biological Sciences, Beijing

\#7 Science Park Rd

Zhongguancun Life Science Park

Beijing, China, 102206

Telephone: +86-10-80728593

Fax: +86-10-80728046

E-mail: shaofeng@nibs.ac.cn 


\section{Abstract}

The inflammasomes are emerging cytosolic defenses against bacterial infections. The inflammasomes converge on inflammatory caspases activation that triggers pyroptosis, and interleukin-1 $\beta / 18$ maturation in the case of caspase-1 activation. The inflammasomes not only detect major bacterial molecules but also sense bacterial virulence activity. Among the canonical caspase-1-activating inflammasomes, the NAIP subfamily of NLR proteins serves as the receptors for bacterial flagellin and type III secretion apparatus; Pyrin indirectly senses Rho modification/inactivation by various bacterial agents; NLRP1 in mice/rats detects the protease activity of anthrax lethal toxin by serving as its substrate. Caspase- 11 and caspase- $4 / 5$ directly recognize bacterial LPS and then become activated. Inflammasome sensing of cytosolic bacteria employs much more diversified biochemical mechanisms, compared with Toll-like receptors-mediated recognition on the membrane. 


\section{Introduction}

The inflammasome is critical for cytosolic defense against infection [1]. The inflammasome activates caspase- 1 by inducing its autoproteolytic processing. Active caspase-1 triggers macrophage pyroptosis and interleukin (IL)-1 $\beta / 18$ maturation for clearing infection. For inflammasome assembly, a pattern recognition receptor (PRR), very often a nucleotide-binding domain (NBD), leucine-rich repeat (NLR) protein, is postulated to recognize a pathogen-associated molecular pattern (PAMP). The process often involves the PYRIN domain (PYD)-caspase activation and recruitment domain (CARD) adaptor ASC that links the NLR to caspase-1. Caspase-1 belongs to the inflammatory caspase group that also includes human caspase- $4 / 5$ and murine caspase-11, all containing an N-terminal CARD. Recent discovery of caspase-11 functioning in detecting Gram-negative bacteria has led to classification of canonical and noncanonical inflammasomes for activating caspase-1 and caspase-11, respectively. Active caspase- 1 and caspase- 11 both trigger pyroptosis by cleaving the newly identified Gasdermin D (GSDMD) protein [2-3].

Key to understanding inflammasome-mediated defense is to identify the PRRs for major bacterial signals and elucidate their mechanism of action. In this review, we discuss how various bacterial products and toxins are sensed by the canonical NAIP/NLRC4, Pyrin and NLRP1 inflammasomes as well as the "noncanonical inflammasome". The NLRP3 and AIM2 inflammasomes (not dedicated to detecting bacteria) are not covered due to space limitation. We emphasize the diverse biochemical strategies employed by the inflammasomes to sense various bacterial signals.

\section{Direct recognition of bacterial structural components}

\section{Recognition of flagellin and type III secretion apparatus by the NAIPs}

Many Gram-negative bacteria harbor a type III secretion system (T3SS) that 
injects virulence effectors into host cells. The T3SS evolved from bacterial flagella and structurally resembles the flagellar export system. Assembly of a functional T3SS critically requires a rod and a needle component. The rod protein polymerizes into a ring structure in the bacterial periplasm but can reach host cytosol by erroneous type III secretion or other nonspecific means; the highly conserved needle protein forms the translocation channel appended to the bacterial surface. While the flagella are prevalent in both pathogenic and nonpathogenic bacteria, the T3SS mainly exists in pathogenic bacteria. Flagellin, the monomeric subunit of flagella, is a major target of host innate immunity. The membrane-bound Toll-like receptor (TLR5) recognizes extracellular flagellin to induce cytokine transcription. Genetic studies of Legionella pneumophila evasion of macrophage immunity suggest a requirement of flagellin for caspase-1-mediated pyroptosis [4-5]. Flagellin entering into host cytosol from the bacteria can trigger caspase-1 activation, which also requires NLRC4 [6-7], an NLR protein known to mediate Salmonella typhimurium-induced caspase-1 activation [8]. Miao et al. further observed that NLRC4 could also sense the rod component of the T3SS in S. typhimurium [9].

How are cytosolic flagellin and the T3SS rod protein detected by the inflammasome? Two studies independently identified the NAIP subfamily of NLRs as the responsible receptors [10-11]. The mouse encodes seven homologous NAIPs (NAIP1-7) while humans only have one (hNAIP). In mice, flagellin is recognized by NAIP5 and its closest paralog NAIP6, which stimulates a physical association of NAIP5/6 with NLRC4; the two NLRs then co-oligomerize to form a large complex for caspase-1 activation (Figure 1). The T3SS rod protein is detected similarly by the NAIP2 receptor. Human monocytes do not respond to cytosolic flagellin and the rod protein, but are sensitive to the T3SS needle protein [11-12]. hNAIP directly binds to 
the needle protein to induce caspase- 1 activation. This inflammasome pathway operates similarly in mice with NAIP1 being the receptor [12-13]. Recognition of T3SS rod/needle proteins by the NAIPs activates caspase-1 through NLRC4, similar to the flagellin-NAIP5/6-NLRC4 inflammasome (Figure 1).

The needle and rod proteins are structurally similar, featuring a hydrophobic helix hairpin. An analogous structural element is present in flagellin and determines NLRC4 inflammasome activation. Ligand recognition by the NAIP is mediated by sequence regions associated with the NBD rather than the C-terminal leucine-rich repeat (LRR) domain [14]. Crystal structure of NLRC4 $\triangle$ CARD shows an autoinhibitory conformation, similar to Apaf-1 in apoptosis [15]; ligand binding likely induces conformational changes of the NAIP and NLRC4 that are then assembled into an inflammasome complex through NBD-mediated oligomerization $[11,14,16]$. A role of infection-induced NLRC4 phosphorylation has been suggested in this activation process [17]. The NAIP-NLRC4 inflammasome has a dominant role in macrophage sensing of various bacterial infections including S. typhimurium and Shigella flexneri. A recent study in mice also suggests an epithelium-intrinsic NAIP-NLRC4 inflammasome that limits S. typhimurium intraepithelial proliferation [18].

\section{LPS recognition by inflammatory caspase-11/4/5}

LPS (lipopolysaccharide) is the major component of Gram-negative bacterial cell wall. For a long time, understanding of innate recognition of LPS was limited to its membrane receptor TLR4/MD2-stimulated cytokine transcription. Recent studies of caspase-11 function suggest a role in sensing Gram-negative bacteria of both pathogenic and non-pathogenic nature [19-23]. Cytosolic LPS is indeed the responsible factor for triggering caspase-11-mediated pyroptosis in mouse macrophages [24-25]. Cytosolic delivery of LPS alone is sufficient to activate 
caspase-11, which is independent of TLR4. When primed with TLR3 agonist, Tlr4 $4^{-/}$ and wild-type mice are equally susceptible to LPS-induced sepsis, establishing a critical role of caspase-11 in endotoxic shock. This intracellular LPS sensing requires bacterial escape from the vacuole [23] and is promoted by interferon-inducible GTPase [26-27].

Knowing the existence of intracellular LPS sensing is paradigm-shifting in innate immunity. However, it was unclear whether the pathway operates in human. A recent study shows that human U937 and THP1 monocytes undergo massive pyroptosis following cytosolic delivery of LPS and this effect is mediated by caspase-4 [28]. Caspase-4 and caspase-11 can functionally complement each other in LPS sensing. Whereas caspase-11 is transcriptionally upregulated by interferon or TLR signaling [19,24-25,29] , caspase-4 is constitutively and highly expressed in various cell types including keratinocytes and epithelial cells, rendering these cells highly sensitive to cytosolic LPS. A crucial role for caspase-4 sensing of LPS in polarized epithelial cells has also been suggested recently [30]. These observations urge re-thinking the relevance of mouse endotoxic shock model to Gram-negative bacteria-induced sepsis in humans.

Knowledge established from apoptosis and caspase-1 researches led to a natural belief that a CARD-containing protein acts as an LPS sensor upstream of caspase-11. Shi et al. found that recombinant caspase-4/11 purified from insect cells are monomers while those from E. coli are $~ 600-\mathrm{kDa}$ multimers; the former can undergo oligomerization upon incubation with Gram-negative lysates or LPS [28]. Biochemical analyses revealed a specific and high-affinity binding between caspase-4/11 and LPS (or its active component lipid A). The binding induces caspase-4/11 oligomerization and importantly activation of the caspases. The CARD 
is required and sufficient for LPS binding, which also oligomerizes upon LPS binding. LPS binding-deficient point mutants of caspase-11/4 are inert in LPS-induced oligomerization/activation and cell pyroptosis. Under-acylated lipid A and LPS variants can bind to caspase-4/11, but do not induce high-order oligomerization and cell pyroptosis [24-25,28]. This echoes LPS recognition by human TLR4/MD2 complex. Biochemically, caspase-5 can also bind to LPS and trigger pyroptosis. Thus, caspase-4/5/11 are innate receptors for cytosolic LPS (Figure 1). The combination of a pattern-recognition sensor (the CARD) and an executioner (the protease domain) in a single polypeptide allows for efficient responses to LPS, guarding the cytosol from Gram-negative invaders.

\section{Detection of bacterial virulence activity}

\section{Sensing bacterial modifications of Rho GTPase by Pyrin}

Bacterial pathogens often target Rho GTPases to modulate host actin cytoskeleton for virulence purposes. Studies have identified numerous Rho-targeting bacterial toxins and effectors that covalently modify Rho GTPases or modulate their nucleotide cycling [31]. Among them, toxin A/B from Clostridium difficile (TcdA/B) inactivate Rho by monoglucosylation of a threonine (Thr-37 in RhoA) [32], which is crucial for $C$. difficile pathogenesis [33]. Xu et al. recently discovered that the glucosyltransferase activity of TcdB could induce caspase-1 activation in mouse and human macrophages [34]. This activation is absent in $A s c^{-/-}$cells, suggesting a requirement of a PYD-containing sensor. Reconstitution of TcdB-induced ASC speck formation in 293T cells led to the identification of Pyrin. Genetic ablation of Pyrin could completely block TcdB-induced inflammasome activation, uncovering the long sought physiological function of Pyrin in innate immunity. Consistently, Pyrin is expressed in innate immune cells and its overexpression can induce ASC-dependent 
caspase-1 activation [35-36]. Gain-of-function mutation in Pyrin-encoding gene $M E F V$ causes familial Mediterranean fever (FMF), a common autoinflammatory disease [37].

The T3SS effector VopS from Vibrio parahaemolyticus and Histophilus somni IbpA also modify Thr-37 (in RhoA) or the nearby Tyr-34 by adenylylation. The $C$. botulinum $\mathrm{C} 3$ toxin ADP-ribosylates Asn-41 in RhoA with a high selectivity for the Rho subfamily. These toxins all modify the GTP-binding switch I region, leading to Rho inactivation. When delivered into macrophages, VopS, IbpA and C3 toxin all can induce caspase-1 activation mediated by the Pyrin/ASC inflammasome [34]. Moreover, Burkholderia cenocepacia can also inactivate Rho GTPases [38-39], resulting from a putative type VI secretion system (T6SS) effector-mediated deamidation of Asn-41 (in RhoA) [34]. Consistently, B. cenocepacia activates the Pyrin inflammasome with severe inflammation in the lung of infected mice. Thus, the Pyrin inflammasome detects diverse modifications-induced Rho inactivation and can potentially cope with a wide spectrum of bacterial infections (Figure 1).

Pyrin does not interact directly with Rho or the modified GTPase. It is most possible that dysfunctioning of the actin cytoskeleton caused by Rho modification triggers Pyrin activation [34]. This model is supported by a recent follow-up study [40]. Mice homozygous for a hypomorphic allele of $W d r 1$ (encoding an actin-depolymerizing cofactor) exhibit spontaneous autoinflammatory disease and thrombocytopenia. The autoinflammation mainly results from Pyrin inflammasome activation [40]. Thus, Pyrin likely senses pathological perturbation of the actin polymerization dynamics. It is worth noting that the actin polymerization-inhibiting agents, cytochalasin D and the actin crosslinking domain of Vibrio cholerae RTX toxin, can not induce Pyrin activation in mouse primary bone macrophages. This 
suggests that Pyrin is not sensitive to all types of actin cytoskeleton disruption and instead detects a specific signaling event of non-physiological nature.

\section{Sensing the metalloprotease activity of anthrax lethal toxin by NLRP1B}

Lethal toxin (LT), a key virulence determinant of Bacillus anthracis, contains the lethal factor (LF) subunit and protective antigen (for receptor binding and endocytic delivery of the holotoxin). LF is a metalloprotease that cleaves mitogen-activated protein kinase kinases (MAPKKs) and blocks MAPK-mediated defenses [41]. LT triggers caspase- 1 activation and pyroptosis in mouse macrophages, requiring its protease activity [42-43]. LT-induced killing only occurs in macrophages of certain genetic background [41], and this allows for positional cloning of mouse Nlrp1b [44]. The polymorphism in rat Nlrpl also determines rat macrophage sensitivity to LT intoxification [45].

NLRP1B is the mouse homolog of NLRP1 in human and rat. The Nlrplb locus in mice has two parologs Nlrpla and a pseudogene Nlrplc. It is now well accepted that NLRP1B and rat NLRP1 serve as the inflammasome sensor for LT protease activity (Figure 1). The NLRP1 subfamily all contains a function to find domain (FIIND) and a CARD following the NBD domain. Different from human NLRP1, the N-termini of mouse and rat NLRP1 lack a PYD or CARD. Sequence differences in the N-terminal 100 residues in LT-sensitive and -resistant rat NLRP1 correlate with phenotypic differences [45], leading to identification of a putative LT cleavage site in LT-sensitive NLRP1 [46]. Indeed LT could specifically cleave the sensitive rat NLRP1; the cleavage is required for caspase- 1 activation reconstituted in nonmacrophage cells. LT also cleaves the $\mathrm{N}$ terminus of mouse NLRP1B that lacks a canonical cleavage site [47]. By engineering a TEV protease cleavage site into NLRP1B, Chavarria-Smith and Vance demonstrated that proteolytic cleavage in the N terminus of NLRP1B is 
sufficient to initiate inflammasome responses [48].

Different from Pyrin sensing of toxin virulence activity, NLRP1B itself is a substrate of LT protease activity (Figure 1). LT cleavage of NLRP1B likely releases the N-terminal autoinhibitory state of NLRP1B. However, this model can not explain several known observations. LT cleaves NLRP1B from both toxin-sensitive and -resistant mice [47], inconsistent with Nlrplb being the genetic determinant for mouse sensitivity to LT. Secondly, LT killing of macrophages is sensitive to proteasome and $\mathrm{N}$-end rule inhibitors [49-50], but these protein degradation inhibitors do not block LT cleavage of NLRP1B $[46,48]$. Despite the incomplete mechanism, LT does represent another example of inflammasome sensing of bacterial virulence. Given that NLRP1B activation does not require a fixed cleavage site, NLRP1B may also function as an inflammasome sensor for other microbial protease activity [48].

\section{Conclusions and perspectives}

LPS and flagellin are general bacterial PAMPs that are also present in nonpathogenic strains. Inflammasome sensing of LPS and flagellin helps to ensure an effective defense upon cytosol contamination by a bacterium. The T3SS and Rho-modifying toxins are unique to pathogens, and their detection allows the innate immune response to distinguish virulent bacteria from avirulent ones. This is likely significant for immune homeostasis in microflora containing mostly commensals. Pyrin and NLRP1 are unique PRRs that sense bacterial virulence activity. The mode of Pyrin action echoes the guard model in plant innate immunity, in which the disease resistance NB-LRR protein often perceives effector inactivation of its host target [51]. Pyrin appears to guard Rho GTPase or its downstream actin cytoskeleton from pathogen destruction. Different from the NB-LRRs, Pyrin presumably can sense many effectors with Rho-modifying activity, and such feature allows mammals to use a few 
PRRs to deal with a large number of pathogens.

In innate immunity, TLRs adopt a common mechanism to recognize different PAMPs. In contrast, inflammasome sensing of cytosolic bacteria involves diversified biochemical strategies. Sensing of flagellin and the T3SS requires two NLRs with NAIP being a receptor and NLRC4 being a signaling adaptor; Rho-modifying toxins are detected by a non-NLR protein Pyrin; LPS is recognized by inflammatory caspases harboring both the sensor and executioner function. Other new or unexpected mechanisms for sensing cytosolic bacteria will likely appear in future studies. Despite multiple and diverse inflammasomes, a certain inflammasome often dominate over others in detecting a particular bacterium. For example, caspase-11 detection of LPS is sufficient to protect mice from lethal challenge with $B$. pseudomallei [23]. Meanwhile, the physiological and pathogenic diversity among bacteria provide a valuable toolbox for discovering new sensing mechanisms. Common to inflammasome-mediated defenses is the activation of inflammatory caspases, but how inflammatory caspases induce pyroptosis remains completely unknown.

\section{Acknowledgements}

We apologize to colleagues whose work could not be cited due to space limitation.

We thank M. Shi for preparing the artwork. Work in the authors' laboratory was supported in part by an International Early Career Scientist grant from the Howard Hughes Medical Institute to F.S. The work was also supported by the National Basic Research Program of China 973 Programs, the China National Science Foundation grant, and the Strategic Priority Research Program of the Chinese Academy of Sciences (XDB08020202) to F.S. 


\section{References and recommended reading}

Papers of particular interest, published within the period of review, have been highlighted as:

* of special interest

** of outstanding interest

1. Lamkanfi M, Dixit VM: Inflammasomes and their roles in health and disease. Annu Rev Cell Dev Biol 2012, 28:137-161.

2. Shi J, Zhao Y, Wang K, Shi X, Wang Y, Huang H, Zhuang Y, Cai T, Wang F, Shao F: Cleavage of GSDMD by inflammatory caspases determines pyroptotic cell death. Nature 2015, doi: 10.1038/nature15514. [Epub ahead of print]

3. Kayagaki N, Stowe IB, Lee BL, O'Rourke K, Anderson K, Warming S, Cuellar T, Haley B, Roose-Girma M, Phung QT, et al.: Caspase-11 cleaves gasdermin D for non-canonical inflammasome signaling. Nature 2015, doi: 10.1038/nature15541. [Epub ahead of print].

** These two studies (References 2 and 3) for the first time identify GSDMD (Gasdermin D) as a functional substrate for caspase-1 and caspase-4/5/11 in pyroptosis. Reference 2 further suggests that other Gasdermin-family members also function as pyroptosis-inducing factors in immune defenses that do not involve inflammatory caspases.

4. Ren T, Zamboni DS, Roy CR, Dietrich WF, Vance RE: Flagellin-deficient Legionella mutants evade caspase-1- and Naip5-mediated macrophage immunity. PLoS Pathog 2006, 2:e18.

5. Molofsky AB, Byrne BG, Whitfield NN, Madigan CA, Fuse ET, Tateda K, Swanson MS: Cytosolic recognition of flagellin by mouse macrophages restricts Legionella pneumophila infection. J Exp Med 2006, 203:1093-1104.

6. Miao EA, Alpuche-Aranda CM, Dors M, Clark AE, Bader MW, Miller SI, Aderem A: Cytoplasmic flagellin activates caspase-1 and secretion of interleukin 1beta via Ipaf. Nat Immunol 2006, 7:569-575.

7. Franchi L, Amer A, Body-Malapel M, Kanneganti TD, Ozoren N, Jagirdar R, Inohara N, Vandenabeele P, Bertin J, Coyle A, et al.: Cytosolic flagellin requires Ipaf for activation of caspase-1 and interleukin 1beta in salmonella-infected macrophages. Nat Immunol 2006, 7:576-582.

8. Mariathasan S, Newton K, Monack DM, Vucic D, French DM, Lee WP, Roose-Girma M, Erickson S, Dixit VM: Differential activation of the inflammasome by caspase-1 adaptors ASC and Ipaf. Nature 2004, 430:213-218.

9. Miao EA, Mao DP, Yudkovsky N, Bonneau R, Lorang CG, Warren SE, Leaf IA, Aderem A: Innate immune detection of the type III secretion apparatus through the NLRC4 inflammasome. Proc Natl Acad Sci U S A 2010, 107:3076-3080.

10. Kofoed EM, Vance RE: Innate immune recognition of bacterial ligands by NAIPs determines inflammasome specificity. Nature 2011, 477:592-595. 
11. Zhao Y, Yang J, Shi J, Gong Y-N, Lu Q, Xu H, Liu L, Shao F: The NLRC4 inflammasome receptors for bacterial flagellin and type III secretion apparatus. Nature 2011, 477:596-600.

12. Yang J, Zhao Y, Shi J, Shao F: Human NAIP and mouse NAIP1 recognize bacterial type III secretion needle protein for inflammasome activation. Proc Natl Acad Sci U S A 2013, 110:14408-14413.

13. Rayamajhi M, Zak DE, Chavarria-Smith J, Vance RE, Miao EA: Cutting edge: Mouse NAIP1 detects the type III secretion system needle protein. J Immunol 2013, 191:3986-3989.

* References 12 and 13 together show that mouse NAIP1 serves as the receptor for the needle protein of bacterial type III secretion system and stimulates NLRC4-mediated caspase-1 inflammasome activation.

14. Tenthorey JL, Kofoed EM, Daugherty MD, Malik HS, Vance RE: Molecular basis for specific recognition of bacterial ligands by NAIP/NLRC4 inflammasomes. Mol Cell 2014, 54:17-29.

** This study makes an unexpected observation that the sequence regions assocaited with the NBD domain rather than the predicted LRR domain in NAIPs are responsible for binding to the inflammasome agonists.

15. Hu Z, Yan C, Liu P, Huang Z, Ma R, Zhang C, Wang R, Zhang Y, Martinon F, Miao D, et al.: Crystal structure of NLRC4 reveals its autoinhibition mechanism. Science 2013, 341:172-175.

16. Halff EF, Diebolder CA, Versteeg M, Schouten A, Brondijk TH, Huizinga EG: Formation and structure of a NAIP5-NLRC4 inflammasome induced by direct interactions with conserved $\mathrm{N}$ - and C-terminal regions of flagellin. J Biol Chem 2012, 287:38460-38472.

17. Qu Y, Misaghi S, Izrael-Tomasevic A, Newton K, Gilmour LL, Lamkanfi M, Louie S, Kayagaki N, Liu J, Komuves L, et al.: Phosphorylation of NLRC4 is critical for inflammasome activation. Nature 2012, 490:539-542.

18. Sellin ME, Muller AA, Felmy B, Dolowschiak T, Diard M, Tardivel A, Maslowski KM, Hardt WD: Epithelium-Intrinsic NAIP/NLRC4 Inflammasome Drives Infected Enterocyte Expulsion to Restrict Salmonella Replication in the Intestinal Mucosa. Cell Host Microbe 2014, 16:237-248.

* This study provides the first in vivo genetic evidences that the NAIP/NLRC4 inflammasome functions in mouse intestinal epithellia to limit bacterial replication.

19. Rathinam VA, Vanaja SK, Waggoner L, Sokolovska A, Becker C, Stuart LM, Leong JM, Fitzgerald KA: TRIF licenses caspase-11-dependent NLRP3 inflammasome activation by gram-negative bacteria. Cell 2012, 150:606-619.

20. Kayagaki N, Warming S, Lamkanfi M, Vande Walle L, Louie S, Dong J, Newton K, Qu Y, Liu J, Heldens S, et al.: Non-canonical inflammasome activation targets caspase-11. Nature 2011, 479:117-121.

21. Akhter A, Caution K, Abu Khweek A, Tazi M, Abdulrahman BA, Abdelaziz DH, 
Voss OH, Doseff AI, Hassan H, Azad AK, et al.: Caspase-11 promotes the fusion of phagosomes harboring pathogenic bacteria with lysosomes by modulating actin polymerization. Immunity 2012, 37:35-47.

22. Case CL, Kohler LJ, Lima JB, Strowig T, de Zoete MR, Flavell RA, Zamboni DS, Roy CR: Caspase-11 stimulates rapid flagellin-independent pyroptosis in response to Legionella pneumophila. Proc Natl Acad Sci U S A 2013, 110:1851-1856.

23. Aachoui Y, Leaf IA, Hagar JA, Fontana MF, Campos CG, Zak DE, Tan MH, Cotter PA, Vance RE, Aderem A, et al.: Caspase-11 protects against bacteria that escape the vacuole. Science 2013, 339:975-978.

24. Hagar JA, Powell DA, Aachoui Y, Ernst RK, Miao EA: Cytoplasmic LPS activates caspase-11: implications in TLR4-independent endotoxic shock. Science 2013, 341:1250-1253.

25. Kayagaki N, Wong MT, Stowe IB, Ramani SR, Gonzalez LC, Akashi-Takamura S, Miyake K, Zhang J, Lee WP, Muszynski A, et al.: Noncanonical inflammasome activation by intracellular LPS independent of TLR4. Science 2013, 341:1246-1249.

** These two studies (references 24 and 25) identify cytosolic LPS as the bacterial factor responsible for the caspase-11 "non-canonical inflammasome" activation. Importantly, this caspase-11 pathway determines endotoxic shock in mice independently of TLR4.

26. Meunier E, Dick MS, Dreier RF, Schurmann N, Kenzelmann Broz D, Warming S, Roose-Girma M, Bumann D, Kayagaki N, Takeda K, et al.: Caspase-11 activation requires lysis of pathogen-containing vacuoles by IFN-induced GTPases. Nature 2014, 509:366-370.

27. Pilla DM, Hagar JA, Haldar AK, Mason AK, Degrandi D, Pfeffer K, Ernst RK, Yamamoto M, Miao EA, Coers J: Guanylate binding proteins promote caspase-11-dependent pyroptosis in response to cytoplasmic LPS. Proc Natl Acad Sci U S A 2014, 111:6046-6051.

28. Shi J, Zhao Y, Wang Y, Gao W, Ding J, Li P, Hu L, Shao F: Inflammatory caspases are innate immune receptors for intracellular LPS. Nature 2014.

** This study makes a totally unexpected discovery that caspase-4/5 as well as their murine orthologue caspase-11, rather than a presumed NLR type of scaffold protein, are intracellular receptors for LPS. The study also provides the first evidences demonstrating that human cells not limited to macrophages and monocytes can sense intracellular LPS.

29. Gurung P, Malireddi RK, Anand PK, Demon D, Vande Walle L, Liu Z, Vogel P, Lamkanfi M, Kanneganti TD: Toll or interleukin-1 receptor (TIR) domain-containing adaptor inducing interferon-beta (TRIF)-mediated caspase-11 protease production integrates Toll-like receptor 4 (TLR4) protein- and Nlrp3 inflammasome-mediated host defense against enteropathogens. J Biol Chem 2012, 287:34474-34483.

30. Knodler LA, Crowley SM, Sham HP, Yang H, Wrande M, Ma C, Ernst RK, 
Steele-Mortimer O, Celli J, Vallance BA: Noncanonical Inflammasome Activation of Caspase-4/Caspase-11 Mediates Epithelial Defenses against Enteric Bacterial Pathogens. Cell Host Microbe 2014, 16:249-256.

* This study shows that the caspase-11 and caspase-4 "non-canonical inflammasome"pathways can sense cytoplasmic LPS in intestinal epithellial cells to control bacterial growth in vivo.

31. Aktories K: Bacterial protein toxins that modify host regulatory GTPases. Nat Rev Microbiol 2011, 9:487-498.

32. Jank T, Aktories K: Structure and mode of action of clostridial glucosylating toxins: the ABCD model. Trends Microbiol 2008, 16:222-229.

33. Kuehne SA, Cartman ST, Heap JT, Kelly ML, Cockayne A, Minton NP: The role of toxin A and toxin B in Clostridium difficile infection. Nature 2010, 467:711-713.

34. Xu H, Yang J, Gao W, Li L, Li P, Zhang L, Gong YN, Peng X, Xi JJ, Chen S, et al.: Innate immune sensing of bacterial modifications of Rho GTPases by the Pyrin inflammasome. Nature 2014, 513:237-241.

** This study for the first time extablishes the Pyrin inflammasome that detects Rho modifications and inactivation by various bacterial toxins with diverse modification activies, including glucosylation by TcdB from $C$. difficile, adenylylation by FIC-domain bacterial effectors, ADP-ribosylation by $\boldsymbol{C}$. botulinum $\mathrm{C} 3$ toxin and deamidation by $B$. cenocepacia. The study shows that the Pyrin inflammasome plays an important role in lung inflammation in $B$. cenocepacia-infected mice.

35. Yu JW, Wu J, Zhang Z, Datta P, Ibrahimi I, Taniguchi S, Sagara J, Fernandes-Alnemri T, Alnemri ES: Cryopyrin and pyrin activate caspase-1, but not NF-kappaB, via ASC oligomerization. Cell Death Differ 2006, 13:236-249.

36. Seshadri S, Duncan MD, Hart JM, Gavrilin MA, Wewers MD: Pyrin levels in human monocytes and monocyte-derived macrophages regulate IL-1beta processing and release. J Immunol 2007, 179:1274-1281.

37. Chae JJ, Cho YH, Lee GS, Cheng J, Liu PP, Feigenbaum L, Katz SI, Kastner DL: Gain-of-function Pyrin mutations induce NLRP3 protein-independent interleukin-1beta activation and severe autoinflammation in mice. Immunity 2011, 34:755-768.

38. Flannagan RS, Jaumouille V, Huynh KK, Plumb JD, Downey GP, Valvano MA, Grinstein S: Burkholderia cenocepacia disrupts host cell actin cytoskeleton by inactivating Rac and Cdc42. Cell Microbiol 2012, 14:239-254.

39. Rosales-Reyes R, Skeldon AM, Aubert DF, Valvano MA: The Type VI secretion system of Burkholderia cenocepacia affects multiple Rho family GTPases disrupting the actin cytoskeleton and the assembly of NADPH oxidase complex in macrophages. Cell Microbiol 2012, 14:255-273.

40. Kim ML, Chae JJ, Park YH, De Nardo D, Stirzaker RA, Ko HJ, Tye H, Cengia L, DiRago L, Metcalf D, et al.: Aberrant actin depolymerization triggers the pyrin 
inflammasome and autoinflammatory disease that is dependent on IL-18, not IL-1beta. J Exp Med 2015, 212:927-938.

** This study analyed a mutant mice with a hereditary autoinflammatory disorder and discovererd that an inactivating mutation in the actin-depolymerizing cofactor Wdr1 causes Pyrin inflammasome activation, which accounts for the hyperautoinflammation in diseased mice. This provides another evidence supporting that Pyrin senses pathological perturbation of the actin polymerization dynamics downstream of Rho GTPases.

41. Turk BE: Manipulation of host signalling pathways by anthrax toxins. Biochem J 2007, 402:405-417.

42. Klimpel KR, Arora N, Leppla SH: Anthrax toxin lethal factor contains a zinc metalloprotease consensus sequence which is required for lethal toxin activity. Mol Microbiol 1994, 13:1093-1100.

43. Cordoba-Rodriguez R, Fang H, Lankford CS, Frucht DM: Anthrax lethal toxin rapidly activates caspase-1/ICE and induces extracellular release of interleukin (IL)-1beta and IL-18. J Biol Chem 2004, 279:20563-20566.

44. Boyden ED, Dietrich WF: Nalp1b controls mouse macrophage susceptibility to anthrax lethal toxin. Nat Genet 2006, 38:240-244.

45. Newman ZL, Printz MP, Liu S, Crown D, Breen L, Miller-Randolph S, Flodman P, Leppla SH, Moayeri M: Susceptibility to anthrax lethal toxin-induced rat death is controlled by a single chromosome 10 locus that includes rNlrp1. PLoS Pathog 2010, 6:e1000906.

46. Levinsohn JL, Newman ZL, Hellmich KA, Fattah R, Getz MA, Liu S, Sastalla I, Leppla SH, Moayeri M: Anthrax lethal factor cleavage of Nlrp1 is required for activation of the inflammasome. PLoS Pathog 2012, 8:e1002638.

47. Hellmich KA, Levinsohn JL, Fattah R, Newman ZL, Maier N, Sastalla I, Liu S, Leppla SH, Moayeri M: Anthrax lethal factor cleaves mouse nlrp1b in both toxin-sensitive and toxin-resistant macrophages. PLoS One 2012, 7:e49741.

48. Chavarria-Smith J, Vance RE: Direct proteolytic cleavage of NLRP1B is necessary and sufficient for inflammasome activation by anthrax lethal factor. PLoS Pathog 2013, 9:e1003452.

* This study shows that artificial cleavage in the $\mathbf{N}$ terminus of an engineered NLRP1B is sufficient to activate the inflammasome. This indicates that cleavage of NLRP1B by anthrax lethal toxin is the mechanism for sensing lethal toxin activity, which does not require other cellular effects of the toxin.

49. Wickliffe KE, Leppla SH, Moayeri M: Killing of macrophages by anthrax lethal toxin: involvement of the N-end rule pathway. Cell Microbiol 2008, 10:1352-1362.

50. Squires RC, Muehlbauer SM, Brojatsch J: Proteasomes control caspase-1 activation in anthrax lethal toxin-mediated cell killing. J Biol Chem 2007, 282:34260-34267.

51. Jones JD, Dangl JL: The plant immune system. Nature 2006, 444:323-329. 


\section{Figure Legend}

\section{Figure 1. Inflammasomes-mediated innate immune detection of cytosolic bacteria and bacterial virulence activity.}

The canonical inflammasomes activate caspase- 1 to induce pyroptosis and IL-1 $\beta / 18$ maturation, which relies on a central scaffold protein (AIM2, NAIPs, Pyrin or NLRP1B) to sense the bacteria. AIM2 is the receptor for dsDNA including that from bacteria while the NAIPs directly recognize bacterial flagellin and components of the toxin-injecting type III secretion apparatus. Pyrin indirectly senses bacterial virulence activities that can covalently modify and inactivate host Rho GTPases; NLRP1B detects the protease activity of anthrax lethal toxin by serving as its substrate. Upon detecting the cognate bacterial signals, these scaffold proteins undergo oligomerization, which often involves adaptor proteins like ASC and/or NLRC4, to assemble the canonical inflammasome complex. For cytosolic LPS, it is directly recognized by caspase-11 in mice and caspase-4/5 in human, resulting in oligomerization and catalytic activation of the caspases. Active caspase-4/5/11 also triggers pyroptosis as well as activation of the canonical NLRP3/ASC inflammasome. These inflammasome pathways employ diverse mechanisms for sensing various bacterial products or virulence activities, playing a critical role in cytosolic defenses against a wide spectrum of bacterial infections. 


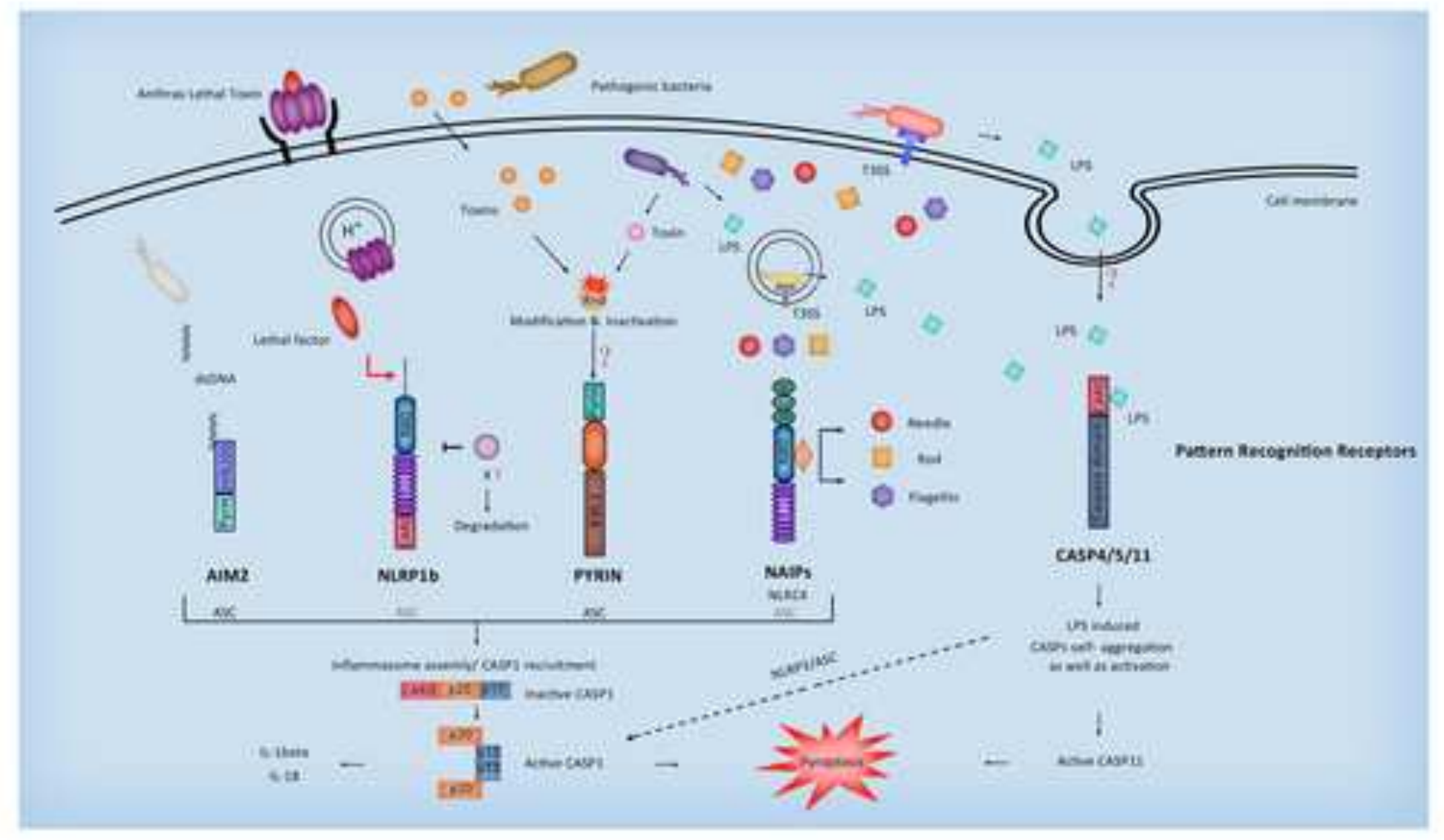

